source of $51.6 \%$ S aureus, and $63.8 \%$ of CNS were isolated from blood cultures. The other strains were from specimens such as wound, catheter, pleural fluid, urine, urethral discharge, eye, and stool (Table).

Resistance rates of $S$ aureus to other commonly used antibiotics were as follows: penicillin, $96.75 \%$; cotrimoxazole, $70.2 \%$; vancomycin, $9.2 \%$; ciprofloxacin, $40.25 \%$; erythromycin, $47.1 \%$; clindamycin, $21 \%$; and gentamicin, $61.03 \%$. Resistance rates for CNS to these antibiotics were $97.82 \%$, $78.3 \%, 4.3 \%, 21.73 \%, 27.7 \%, 10.6 \%$, and $45.65 \%$, respectively.

The ideal method for identification of MRSA is direct detection of $m e c A$, but tests for the gene or its products are not yet performed routinely in most clinical microbiology laboratories. A reliable method for detection of MRSA in routine work is the oxacillin screen agar test. Oxacillin is preferred to methicillin because it is more stable. Sensitivity of oxacillin screen agar approaches $100 \%$ for the detection of MRSA and $95 \%$ for CNS. ${ }^{4}$

In our country, the routine method for susceptibility testing is disk diffusion, but unfor tunately this method is not well-standardized in many laboratories; for this reason, data obtained from various studies are not comparable. In addition, in developing countries such as Iran, standard disks and strains of organism are not available. Delayed transportation and failure of cold storage also affect the quality of antibiotics used as diagnostic reagents. ${ }^{5}$ Our study was the only standard method for detection of MRSA in Iran.

As mentioned before, unfortunately the frequency of MRSA and resistance of CNS to methicillin are very high in Imam Khomeini Hospital. The rate of MRSA may be due to poor quality and misuse of antibiotics, inadequate hospital infection control, and inadequate drug-resistance surveillance.

\section{REFERENCES}

1. Cohen FL, Tartasky D. Microbial resistance to drug therapy: a review. Am J Infect Control 1997;25:51-64.

2. Rubin RI, Harrington CA, Poon A, Dietrich K, Green JA, Moiduddin A. The economic impact of Staphylococcus aureus infection in New York City hospitals. Emerg Infect Dis 1999;5:9-17.

3. Papia G, Louie M, Tralla A, Johnson C, Collins V, Simor AE. Screening highrisk patients for methicillin-resistant Staphylococcus aureus on admission to the hospital: is it cost effective? Infect Control Hosp Epidemiol 1999;20:473-477.

4. Chambers HF. Detection of methicillinresistant staphylococci. Infect Dis Clin North Am 1993;7:425-433.

5. Okeke IN, Lamikanra A, Edelman R. Socioeconomic and behavioral factors leading to acquired bacterial resistance to antibiotics in developing countries. Emerg Infect Dis 1999;5:18-27.

Mohammad Rahbar, PhD
Homayon Babazadeh, MSc
Nosratalh Zarghami, PhD
Urmia University of Medical Sciences
Urmia, Iran

Mohammad Rahbar, PhD

Homayon Babazadeh, MSc Urmia, Iran

\title{
MRSA Infection in Patients With Cystic Fibrosis
}

\section{Gina Pugliese, RN, MS \\ Martin S. Favero, PhD}

Miall and colleagues from Leeds, United Kingdom, note that methicillinresistant Staphylococcus aureus (MRSA) infection is increasingly found in patients with cystic fibrosis (CF). They conducted a study to determine whether MRSA infection has a deleterious effect on the clinical status of children with CF. Children with MRSA in respiratory cultures during a 7year period were identified and compared with controls matched for age, gender, and respiratory function. Respiratory function tests, anthropometric data, ShwachmanKulczycki score, Northern chest radiograph score, intravenous and nebulized antibiotic therapy, and steroid therapy were compared 1 year before and 1 year after MRSA infection.

From a clinical population of 300,10 children had positive sputum or cough swab cultures for MRSA. Prevalence rose from 0 in 1992 through 1994 to 7 in 1998. Eighteen controls were identified. Children with MRSA showed significant worsening of height standard deviation scores and required twice as many courses of intravenous antibiotics as controls after 1 year. They had significantly worse chest radiograph scores at the time of the first MRSA isolate and 1 year later, but showed no increase in the rate of decline in chest radiographic appearance. There was a trend toward lower FEV (1) and FEF (25-75) in children with MRSA.
There were no significant differences between the two groups with respect to change in weight, body mass index, or Shwachman score. There was no significant difference in prior use of steroids or nebulized antibiotics.

The authors concluded that MRSA infection in children with $\mathrm{CF}$ does not affect respiratory function significantly, but may have an adverse effect on growth. Children with MRSA require significantly more courses of intravenous antibiotics and have a worse chest radiograph appearance than controls.

FROM: Miall LS, McGinley NT, Brownlee KG, Conway SP. Methicillinresistant Staphylococcus aureus (MRSA) infection in cystic fibrosis. Arch Dis Child 2001;84:160-162. 


\section{Get the inside story about your patient's catheter.}

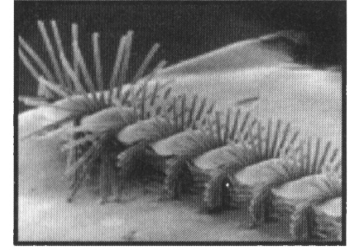

A Scanning electron micrograph of clean brush before use.

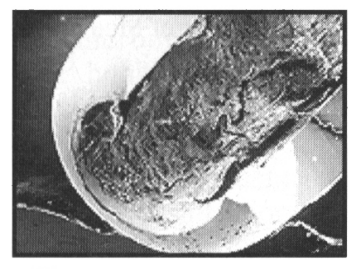

B Single-lumen catheter cut open, exposing biofilm and fibrin.

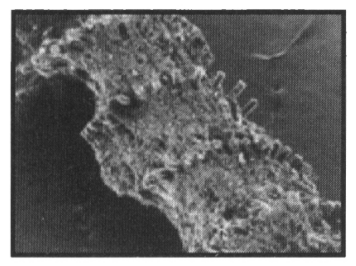

Brush after going down catheter and retrieving biofilm and fibrin sample.

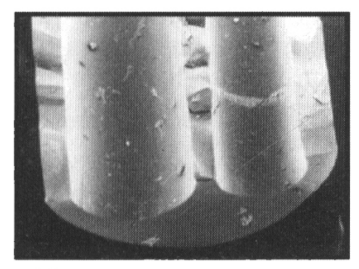

D Double-lumen catheter cut open after being brushed. Bio-load was similar to singlelumen in Photo B.

\section{Preserve your patient's access sites.}

\section{The FAS Endoluminal Brush}

The FAS Endoluminal Brush is a simple, economical, painless solution to detecting catheter infections. It provides the "Inside Story" from your patient's catheter, in situ.

Before you pull a suspect catheter, check for sepsis. The Catheter Brush enables you to get a direct, definitive biofilm and fibrin sample of what's growing inside.

Published studies show $80 \%$ of catheters suspected of infection return a negative culture and do not need replacement.

Use the Catheter Brush to save time, money and discomfort, while improving patient care.

Brush First.

\section{Stat-Lab International, Inc.}

Master distributor for North America and Japan

Call toll free: $(800) 663-4705$

or (206) 254-0820

E-mail: dhatton@stat-lab.com

Photos courtesy of Dr. Peter Kite, R\&D Microbiology, Leeds General Infirmary, UK. 